\title{
Model parameters
}

\begin{tabular}{|c|c|c|}
\hline Parameter & Description & Value \\
\hline$\alpha_{\text {cue }}$ & sensorimotor association learning rate & 0.01 \\
\hline$\alpha_{\text {reward }}$ & expected reward learning rate & 0.005 \\
\hline$\lambda_{1}$ & rise time constant of eligibility traces & 0.01 \\
\hline$\lambda_{2}$ & decay time constant of eligibility traces & 0.004 \\
\hline$\eta_{v i s}$ & visual input gain & 8.0 \\
\hline$\eta_{\text {cue }}$ & cue input gain & 0.1 \\
\hline$\eta_{\text {cost }}$ & cost input gain & -0.1 \\
\hline$\eta_{\text {reward }}$ & expected reward input gain & 0.35 \\
\hline$\eta_{\text {effector }}$ & effector competition input gain & -1.25 \\
\hline$\gamma$ & action threshold & 0.9 \\
\hline$\gamma_{\text {rapid }}$ & action threshold for rapid movements (Figure 3) & 0.7 \\
\hline
\end{tabular}

